\title{
Hypertensive Krise auch ambulant beherrschbar
}

\author{
Patienten mit hypertensiver Krise („hypertensive urgency“) müssen nicht in die Notaufnahme \\ oder stationär in die Klinik, sondern können ambulant behandelt werden.
}

_ In den Jahren 2008-2013 suchten mehr als 2 Millionen Patienten die Kliniken in Cleveland auf. Darunter befanden sich 58.535 (4,5\%) Personen mit einer hypertensiven Krise, also mit einem Blutdruckwert von über $180 \mathrm{mmHg}$ systolisch und/oder $110 \mathrm{mmHg}$ diastolisch, neben dem keine anderweitigen Beschwerden existierten. Der mittlere Blutdruck der Patienten lag bei 183/96 mmHg. 10,2\% von ihnen hatten einen systolischen Blutdruck über $200 \mathrm{mmHg}$, $5,7 \%$ einen diastolischen über 120 $\mathrm{mmHg}$.

Nur 426 Patienten (0,7\%) blieben in der Klinik, während 58.109 entlassen wurden. Die stationär aufgenommenen Patienten hatten einen um 16/11 mmHg höheren Blutdruck, häufiger Nierenerkrankungen und/oder eine Hypertonie in der Vorgeschichte, nahmen aber die gleiche Zahl von Antihypertensiva ein. Die Analyse einer repräsentativen Stichprobe ergab, dass die Zahl schwerer kardiovaskulärer Komplikationen in den folgenden sechs Monaten bei lediglich $0,9 \%$ lag - und zwar sowohl bei Patienten, die in der Klinik blieben, als auch bei solchen, die nach Hause entlassen wurden.

- Patel KK, Young L, Howell EH et al. Characteristics and outcomes of patients presenting with hypertensive urgency in the office setting. JAMA Intern Med. 2016;176:981-8

\section{KOMMENTAR}

Wenn hohe Blutdruckwerte mit zerebralen, kardialen oder okulären Symptomen und Komplikationen einhergehen, besteht ein seltener, bedrohlicher hypertensiver Notfall, der eine sofortige stationäre Aufnahme verlangt. Hohe Blutdruckwerte bei asymptomatischen Patienten, auch solche über $200 \mathrm{mmHg}$ sys- tolisch und $120 \mathrm{mmHg}$ diastolisch, sind dagegen häufig - aber kaum jemals kurzfristig bedrohlich. Die Vorstellung in Notfallambulanzen führt in der Regel zu stationären Aufnahmen und umfangreicher Diagnostik, laut dieser Studie aber nicht zur Verhinderung von Komplikationen oder zu besseren Ergebnissen. Völlig unbefriedigend ist leider das Langzeitergebnis der Untersuchung: Nach sechs Monaten war der Blutdruck bei $66,6 \%$ Patienten, die in der Klinik geblieben waren, und bei $64,6 \%$ der Entlassenen schlecht eingestellt.

Die Diagnose "hypertensive Krise" löst bei Patienten und Ärzten zwar Ängste und Handlungszwänge aus, hat aber kurzfristig eine gute Prognose. Gefordert sind nicht Sofortmaßnahmen, sondern eine geduldige und langfristige Beobachtung und Behandlung des Blutdrucks.

Prof. Dr. med. H. Holzgreve

\section{Dieser Schädel erinnert an einen Igel}

Ein 35-jähriger Mann mit bekannter Sichelzellanämie kam wegen Kopfschmerzen und einer hämolytischen Krise in die Nothilfe. Die Computertomografie des Schädels zeigte eine Verdickung der Kalotte mit radiär angeordneten, stachelförmig aussehenden Verdichtungen der Diploe bei Abbau der Tabula externa und Rarefizierung der Tabula interna.

Das CT erinnert in diesem Fall fast schon an einen Igel, die gängige Beschreibung lautet allerdings „Bürstenschädel“. Sie stammt historisch betrachtet von konventionellen radiologischen Bildern, wenngleich man auch im CT und im MRT ähnliche Konfigurationen erkennen kann. Das charakteristische Bild kommt vor allem bei Patienten mit schwerer Anämie, insbesondere Thalassaemia major und weniger häufig auch bei der Sichelzellanämie vor. Auch bei Patienten mit hämolytischer Anämie, Sphärozytose, chronischer Eisenmangelanämie oder kongenitalen Herzvitien mit Zyanose können ähnliche Veränderungen der Schädelkalotte auftreten. Sie entstehen durch eine kompensatorische Proliferation des roten Knochenmarks. Die
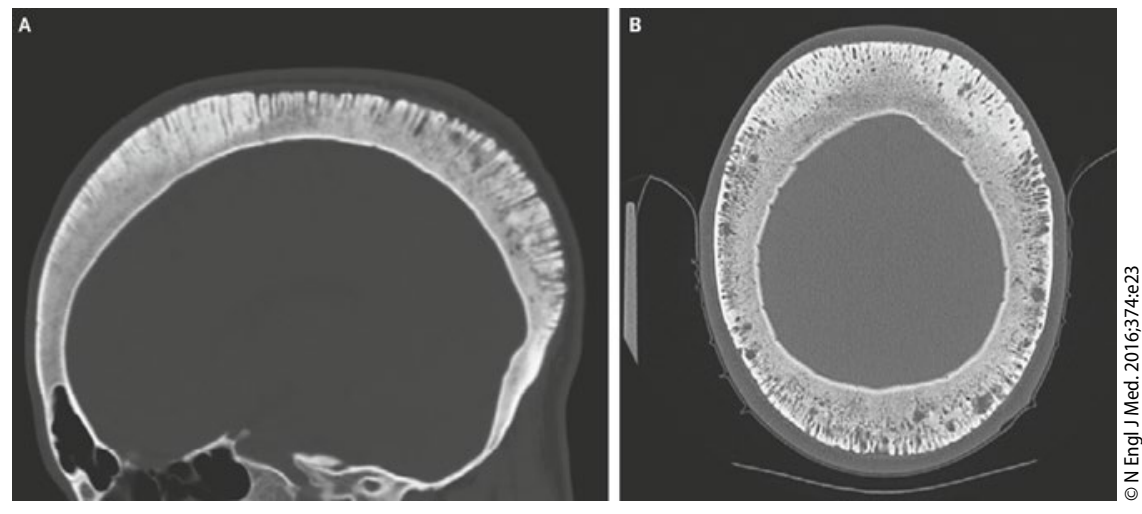

Schädel-CT: Verdickung der Kalotte mit radiär angeordneten, stachelförmig aussehenden Verdichtungen der Diploe.

Zerstörung der Tabula externa mit Ausbildung von Spiculae führt zu dem typischen Bild, das an eine Bürste erinnert.

Prof. Dr. med. H. S. FüeßI

- Martin L, Rackard F (Imartin@ucsd.edu). Hair-on-end sign. N Engl J Med. 2016;374:e23 